\title{
Editorial
}

\section{Cavernous sinus and its role in eye movements and eye health}

\author{
Atul Goel, Manu Kothari* \\ Departments of Neurosurgery and *Anatomy, King Edward Memorial Hospital and Seth G.S. Medical College, Parel, Mumbai, India
}

The cavernous sinus is relatively small in size in human beings when compared to that in lower animals. Like the 'human tail', the general observation is that the cavernous sinus is a vestigial structure. There are only isolated reports in the literature speculating about the possible function of this structure. ${ }^{[1,2]}$

The cavernous sinuses are relatively large in lower animals probably because their function is more pronounced. The eye movements in these animals are not only essential in the provision of binocular vision, but also essential in protection of their lives. For example, when the deer runs and the tiger is running behind in an attempt to catch it, the deer is able to see not only ahead, but can also see the chasing tiger at the same time. This is due to the wide range and rapidity of its eye movements. The cavernous sinuses in the fish are relatively much large in size. The eye movements in the fish not only assist in provision of a wide range of visual field, but are also essential in providing balance whilst swimming. Absence of vision in one eye is not compatible with life in most fishes. The range of eyeball movements and its possible functions in a human are limited and that may be the possible reason of the cavernous sinus being relatively small in size.

The cavernous sinuses drain the entire venous blood of the orbit. Their relative capaciousness, ability to stretch and their interconnectedness are essential elements on which the hydrodynamics of the fluid filled anterior and posterior chambers of the eyeball thrive. The fluid turnover in both the chambers has to be rapid, fairly constant and at highly controlled pressure. A single cavernous sinus provides a large efferent area into which the orbit can quickly drain any excess blood. As a further backup, one cavernous sinus is connected to another to provide yet more area. The sinusoidal dilatation of the venous circuitry in the region of the cavernous sinus appears to be necessary for the performance of the function of a sponge or a shock absorber of the eyeball. The pathos of the eye when the cavernous sinuses are thrombosed speaks for the significant role that they play during physiological states. All eye movements are lost despite functioning and anatomically intact cranial nerves. In severe cavernous sinus thrombosis, the venous drainage from the eye ball can be hampered, and there can be severe rise in intraocular pressure and even blindness. The eye movements and function return to normal when the blood flow within the venous spaces of the cavernous sinus is restored. It appears that the veins participate in all the movements of the eyeball by constant drainage and blood pooling. The intercavernous sinuses probably assist in coordinating movements of both the eyes. When an external pressure is applied on the eyeball, the venous spaces behind the eyeball and the cavernous sinuses protect the eyeball by providing a buffer space, a situation simulating the buffer provided by cerebrospinal fluid in the brain. The rapid drainage that the cavernous sinuses provide protect the delicate retinal artery, which is devoid of muscularis layer, from raised pressure and consequent rupture due to the venous congestion.

\section{References}

1. Goel A. Cavernous sinus: a speculative note. .J Clin Neuroscience 1996;3: 281 2. Goel A, Kothari M, Kobayashi S. Cavernous sinus: a philosophy and anatomy. In: Kobayashi S, Goel A, Hongo K. Neurosurgery of complex tumours and vascular lesions. New York; Churchill Livingstone: 1997. p. 147-162. 